\title{
Differential proteomics and physiology of Pseudomonas putida KT2440 under filament-inducing conditions
}

\author{
Aurélie Crabbé1 ${ }^{1,4,5}$, Baptiste Leroy², Ruddy Wattiez ${ }^{2}$, Abram Aertsen³ ${ }^{3}$ Natalie Leys ${ }^{1}$, Pierre Cornelis ${ }^{4}$
} and Rob Van Houdt ${ }^{*}$

\begin{abstract}
Background: Pseudomonas putida exerts a filamentous phenotype in response to environmental stress conditions that are encountered during its natural life cycle. This study assessed whether $P$. putida filamentation could confer survival advantages. Filamentation of $P$. putida was induced through culturing at low shaking speed and was compared to culturing in high shaking speed conditions, after which whole proteomic analysis and stress exposure assays were performed.

Results: $P$. putida grown in filament-inducing conditions showed increased resistance to heat and saline stressors compared to non-filamented cultures. Proteomic analysis showed a significant metabolic change and a pronounced induction of the heat shock protein IbpA and recombinase RecA in filament-inducing conditions. Our data further indicated that the associated heat shock resistance, but not filamentation, was dependent of RecA.

Conclusions: This study provides insights into the altered metabolism of $P$. putida in filament-inducing conditions, and indicates that the formation of filaments could potentially be utilized by $P$. putida as a survival strategy in its hostile, recurrently changing habitat.
\end{abstract}

Keywords: Pseudomonas putida KT2440, Filamentation, Elongation, SOS response, RecA, Shaking speed, Stress resistance

\section{Background}

The soil bacterium Pseudomonas putida has to cope with diverse and variable habitat-associated stressors to ensure its survival [1]. Besides the exposure of P. putida to toxic pollutants and antibacterial compounds in soils, this bacterium encounters osmotic, thermal, oxidative and starvation stresses in the natural habitat [2-5].

Under certain laboratory growth conditions, $P$. putida exerts a filamented phenotype [6]. Filamentation occurs due to the lack of septation during the cell growth process and results in the formation of elongated bacteria, which is typically a consequence of DNA damage or envelope stress [7]. Cell division inhibition is most commonly mediated by the DNA-damage response system (SOS

\footnotetext{
* Correspondence: rob.van.houdt@sckcen.be

'Unit of Microbiology, Expert Group Molecular and Cellular Biology, Institute for Environment, Health and Safety, Belgian Nuclear Research Centre (SCK (EN), Mol, Belgium

Full list of author information is available at the end of the article
}

response) [7]. DNA damage (for example, due to ultraviolet irradiation or oxidative radicals) results in the exposure of single-stranded DNA stretches that become covered by the RecA recombinase. In this nucleoprotein filament, RecA becomes activated and stimulates the autoproteolysis of the LexA repressor, which in turn results in derepression of the SOS regulon. While most of the SOS genes are involved in DNA-repair, some carry out other functions, such as the inhibition of cell division. In this context, SulA (which is regulated by LexA) physically inhibits FtsZ polymerization and causes the formation of non-septated bacterial filaments, in order to prevent transmission of damaged DNA to daughter cells. In absence of SOS induction, however, direct chemical inhibition of FtsZ can also lead to bacterial elongation [8].

While reports describing conditions that induce $P$. putida filamentation are scarce, filamentation of other bacteria has been shown in response to DNA damage (as 
described above), nutrient deprivation, low temperature, media composition, low shaking speed and high osmolarity [6,9-11]. Additionally, the different stages of biofilm development in P. putida have been associated with alterations in bacterial length [12]. Furthermore, the plant-produced alkaloid berberine was found recently to induce filamentation in Escherichia coli K12 [8]. Collectively, these studies indicate that conditions and/or products encountered by P. putida during its natural life cycle could induce filamentation.

For a variety of (opportunistic) pathogens, the filamentous morphology has been shown to provide survival advantages [7]. More specifically, uropathogenic Escherichia coli (UPEC) filaments were more proficient in evading neutrophil phagocytosis compared to nonfilamented UPEC [13]. UPEC filamentation was presumably induced in response to effectors of the host innate immunity. The intracellular survival of Salmonella enterica serovar Typhimurium in macrophages in vitro is also associated with a filamentous phenotype, which is probably induced by macrophage production of nitric oxide radicals [14]. In addition, filamentation has been shown to play a role in the infection process of, among others, Proteus mirabilis, Legionella pneumophila, Mycobacterium tuberculosis and Shigella flexneri [7].

It remains unclear which mechanisms are at the origin of $P$. putida filamentation, which metabolic changes occur in $P$. putida filaments, and whether the $P$. putida filamented phenotype could confer environmentally advantageous traits. This study is the first to assess the global proteome and stress resistance of $P$. putida KT2440 when grown in conditions that induce filamentation.

\section{Results}

Morphologic and growth analysis of $P$. putida KT2440 grown in filament and non-filament inducing conditions The formation of filaments by $P$. putida KT2440 cultures was induced by overnight shaking at low speed (i.e., 50 rpm) [6], and corroborated by microscopic and flow cytometry analysis (Figure 1A and C). A bacterial culture shaken at high speed (i.e., $150 \mathrm{rpm}$ ) was used as a nonfilamentous control (Figure $1 \mathrm{~B}$ and D). Figure 1 demonstrates a clear difference in population heterogeneity between $50 \mathrm{rpm}$ and $150 \mathrm{rpm}$-grown P. putida KT2440, with $50 \mathrm{rpm}$-grown bacteria showing an increased size distribution (based on forward scatter). The increase in bacterial size for $50 \mathrm{rpm}$-grown $P$. putida is also reflected in the comparative flow cytometry histogram (Figure 1E). Nucleic acid staining of $50 \mathrm{rpm}$ and $150 \mathrm{rpm}$-grown bacteria (Figure $1 \mathrm{C}$ and $\mathrm{D}$ ) confirmed the size differences. In order to rule out any effects of differences in growth phase between the two test conditions, the growth of P. putida KT2440 as a function of shaking speed was determined (Figure 2). No statistically significant $(\mathrm{p}<0.05)$ differences were found, only a slight significant increase in cell numbers was observed at $6 \mathrm{~h}$ for the $150 \mathrm{rpm}$-grown cultures. In agreement with the OD measurements, no statistically significant $(\mathrm{p}<0.05)$ differences were observed at 15 $\mathrm{h}$ in viable counts nor in biomass $(45.3 \pm 1.6 \mathrm{mg}$ wet weight $/ 5 \mathrm{~mL}$ for 50 -rpm and $44.1 \pm 0.9 \mathrm{mg}$ weight $/ 5 \mathrm{~mL}$ for 150-rpm cultures). As differences in the dissolved oxygen concentrations are expected to occur at different shaking speeds, the dissolved oxygen was measured for $50 \mathrm{rpm}$ and $150 \mathrm{rpm}$-grown bacteria as a function of culture time. As presented in Figure 2, $50 \mathrm{rpm}$ cultures reached undetectable oxygen levels after approximately $1.75 \mathrm{~h}$, while this was only after $4 \mathrm{~h}$ for $150 \mathrm{rpm}$. Further, the maximum oxygen transfer rate at $150 \mathrm{rpm}$, calculated based on [15], was approximately 2.5 times higher than at $50 \mathrm{rpm}$.

\section{Stress resistance of $P$. putida KT2440 grown in filament and non-filament inducing conditions}

The stress resistance of $P$. putida KT2440 grown in filament-inducing and non-filament-inducing conditions (15 hours of growth) was investigated. P. putida KT2440 grown at $50 \mathrm{rpm}$ demonstrated an increased resistance to heat shock (12.5-fold, $\mathrm{p}=0.003)$ and saline stress $(2.1$-fold, $\mathrm{p}=0.005)$, when compared to cells grown at $150 \mathrm{rpm}$ (Figure 3). The acid stress resistance profile was similar for cultures grown at both tested shaking speeds.

\section{Proteomic analysis of $P$. putida KT2440 grown in filament and non-filament inducing conditions}

In order to investigate the molecular basis of the observed increased stress resistance of $P$. putida KT2440 grown in filament-inducing conditions, differential proteomic analysis was performed on samples after 15 hours of growth. This time point was chosen with the aim of obtaining an accumulation of effects associated with cultivating at different shaking speeds. Two biological replicates were analyzed, using a post-digest ICPL protocol, allowing the identification of 659 unique proteins, of which 542 were quantified. Subcellular localization prediction using PSORTb revealed that identified proteins mainly belonged to the cytoplasmic compartment and cytoplasmic membrane (Figure 4A). Almost 300 proteins could be quantified in both biological replicates and the calculated correlation between the 2 datasets reached 0.89 , suggesting a very high reproducibility of our observations (Figure 4B). Finally, among the 542 quantified proteins, 223 proteins had a fold change lower than 0.66 or higher than 1.5 revealing that the difference in shaking speed had a major influence on the proteome of P. putida KT2440. The heat shock protein IbpA was induced the most in filament-inducing conditions (8.33 fold), followed by periplasmic phosphate-binding proteins (PP_2656, 4.26 fold; PP_5329, 3.33 fold). The RecA protein was induced 2.35 fold (Table 1). Among the differentially regulated proteins, a majority was involved in metabolic activity (Table 1). Altered metabolic activity in P. putida 


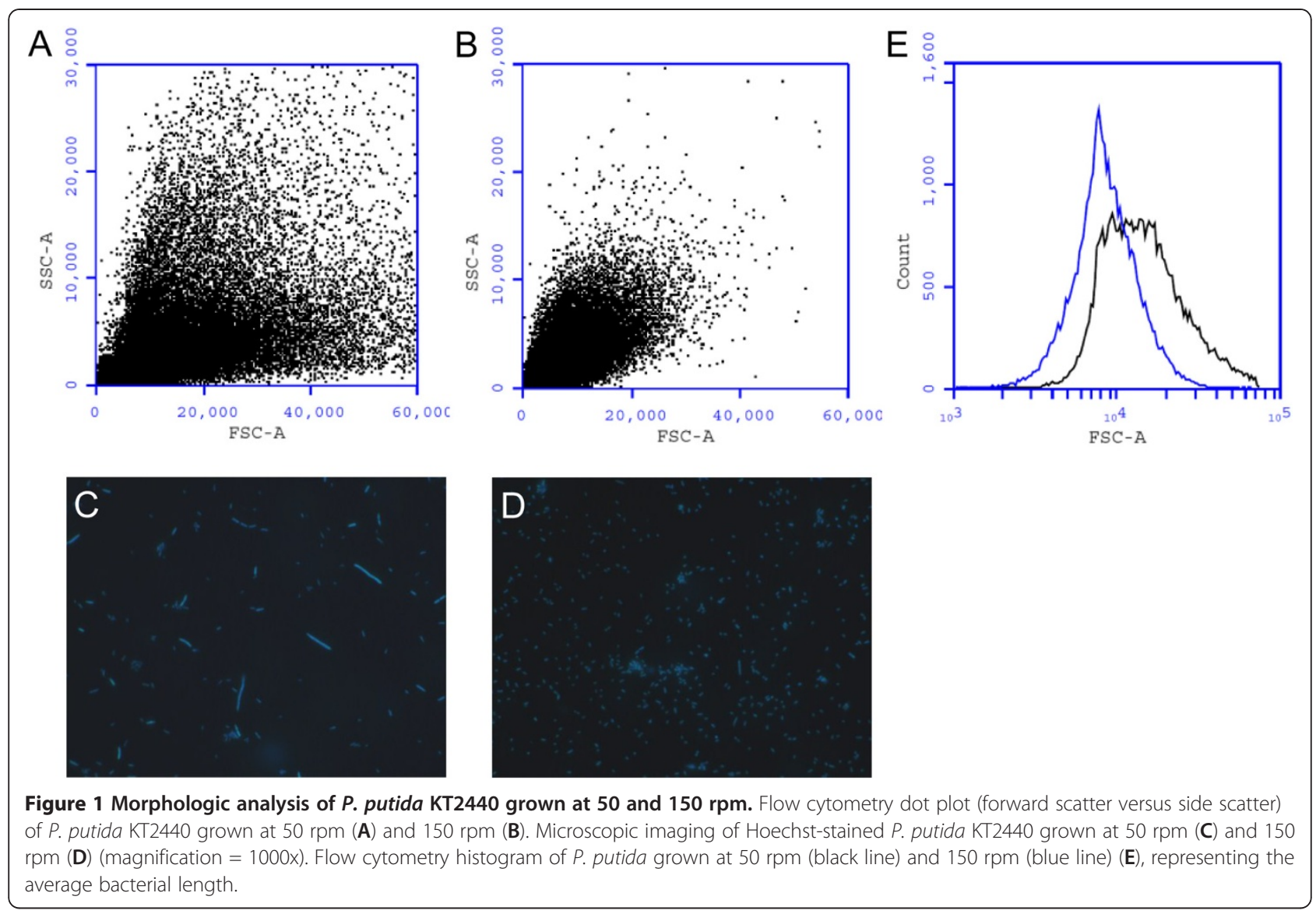

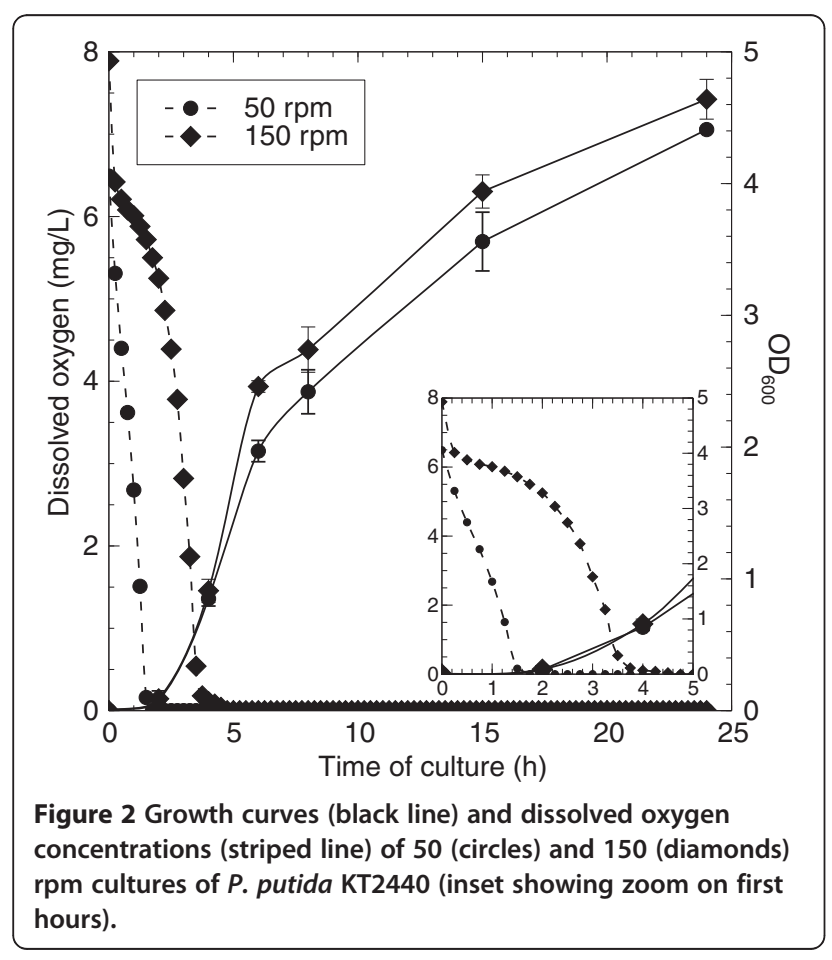

filaments was reflected in (i) down-regulation of a protein involved in purine/pyrimidine catabolism (PP_4038, 0.26 -fold), (ii) down-regulation of proteins involved in the degradation of allantoate (PP_4034, 0.38-fold) and formation/downstream catabolism of urea (PP_0999, 0.23-fold; PP_1000, 0.28-fold; PP_1001, 0.24-fold) and glyoxylate (PP_4116, 0.27-fold; PP_2112, 0.42-fold and PP_4011, 0.25-fold), (iii) down-regulation of proteins involved in the production of ATP (PP_1478, 0.23-fold; PP_0126, 0.37-fold and PP_1478, 0.23-fold), (iv) differential expression of proteins involved in the metabolism of amino acids (PP_4666, 0.24-fold; PP_4667, 0.28-fold; PP_3433, 0.25-fold and PP_4490, 0.47-fold). In addition, proteomic analysis of $P$. putida filaments indicated downregulation of formate metabolism (PP_0328, 0.38-fold), lipid degradation (PP_3282, 0.21-fold) and synthesis of polyhydroxyalkanoate (PP_5007, 0.33-fold).

\section{Role of RecA in P. putida KT2440 filamentation and stress} resistance

The increased abundance of RecA (PP_1629, 2.35-fold) in $50 \mathrm{rpm}$ cultures of $P$. putida KT2440 (Table 1) suggested the activation of the SOS response. Since only induction of RecA was observed, this could indicate a 

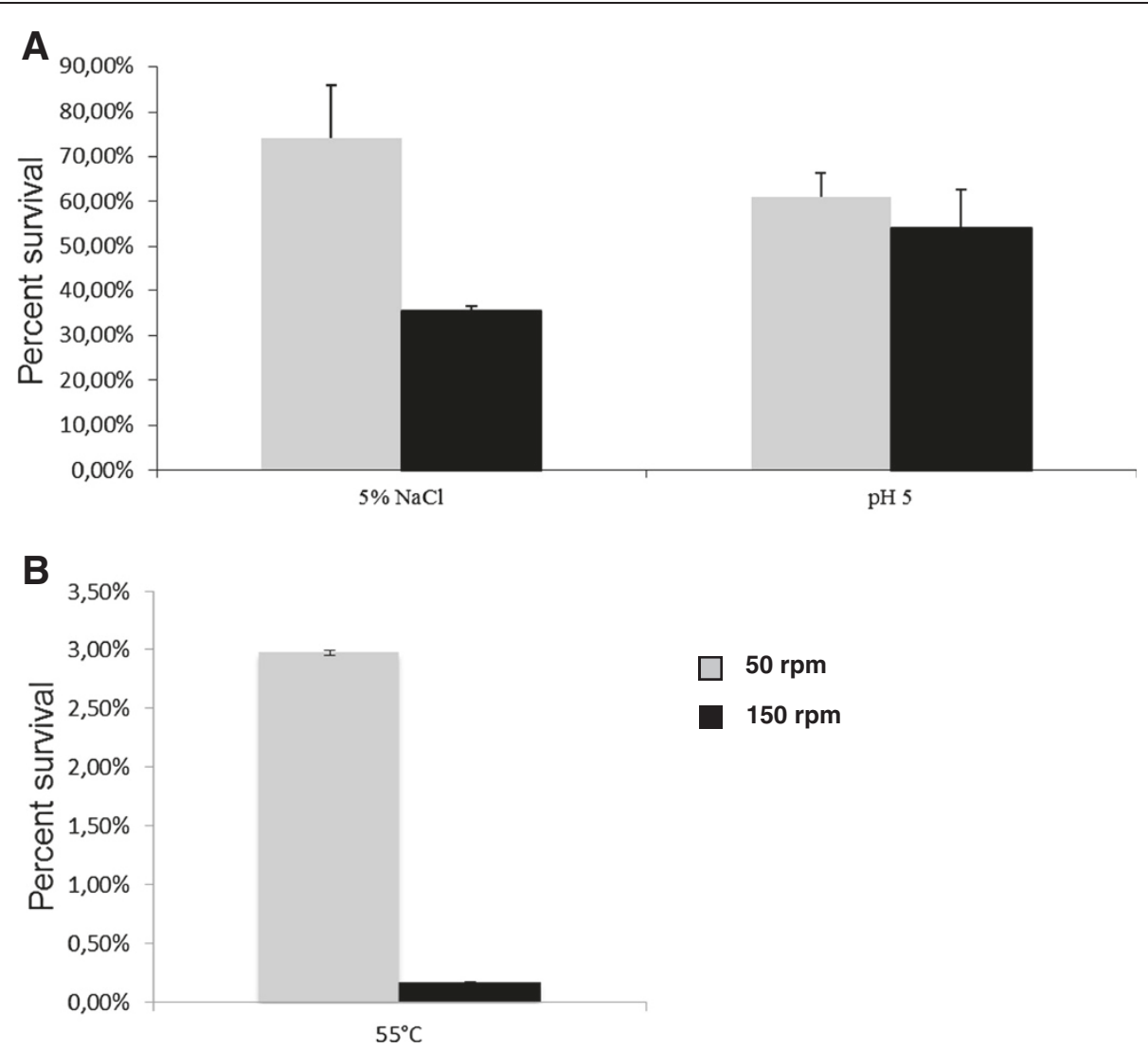

Figure 3 Resistance profile of $P$. putida $\mathrm{KT} 2440$ exposed to $5 \% \mathrm{NaCl}$ and $10^{-4} \mathrm{M}$ citric acid (A), and $55^{\circ} \mathrm{C}$ (B) for 30 min following growth at 50 and $150 \mathrm{rpm}$.

mild SOS response [16]. In addition, the heterogeneity of the SOS response at single cell level could be masked at the population level [17]. This heterogeneity was also apparent in cell morphology between $50 \mathrm{rpm}$ - and 150 rpm-grown $P$. putida KT2440 (Figure 1). In order to determine whether $50 \mathrm{rpm}$-induced filamentation in $P$. putida KT2440 was indeed dependent on RecA, an isogenic rec $A$ mutant cultured in 50 and $150 \mathrm{rpm}$ conditions was examined. Intriguingly, the $50 \mathrm{rpm}$-grown $P$. putida KT2440 recA mutant filamented at similar levels as the wild type $P$. putida KT2440 (Additional file 1: Figure S1). In contrast to filamentation, the increased heat shock resistance of $P$. putida KT2440 grown at $50 \mathrm{rpm}$ was RecA-dependent, since an isogenic recA mutant was equally resistant to heat shock when grown at $50 \mathrm{rpm}$ or 150 rpm (Additional file 2: Figure S2).

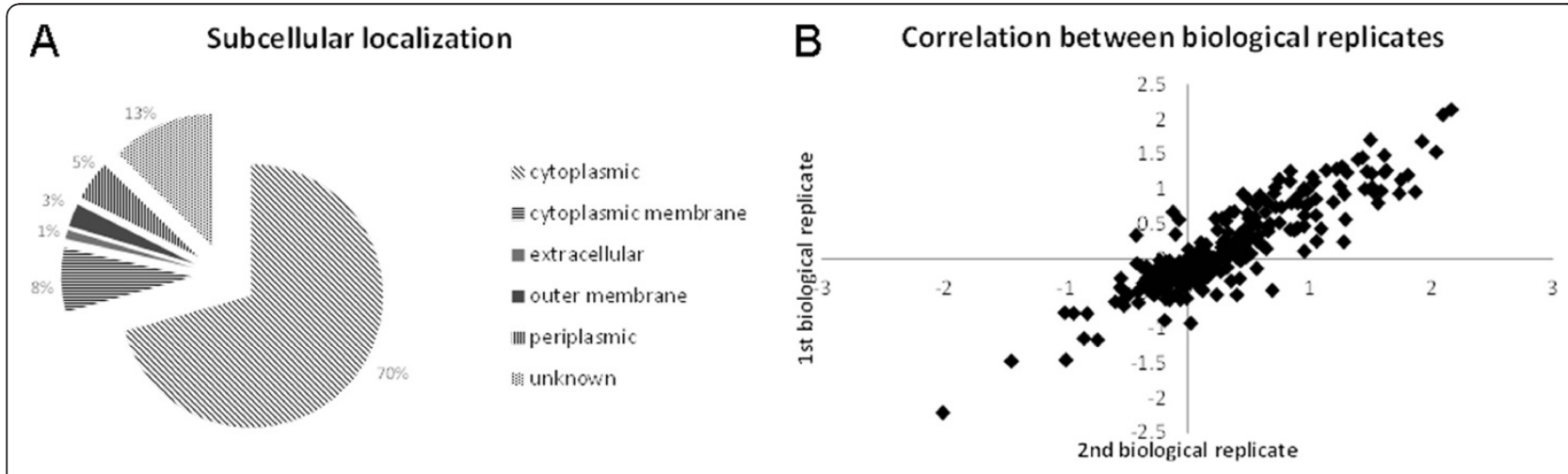

Figure 4 Subcellular localization prediction using PSORTb revealed that identified proteins mainly belong to cytoplasmic compartment and cytoplasmic membrane (A). Correlation between fold changes reached 0.89 which suggest high reproducibility of the proteomic data (B). 
Table 1 Comparative proteome profile of $P$. putida grown at $50 \mathrm{rpm}$ and $150 \mathrm{rpm}$

\begin{tabular}{|c|c|c|c|c|}
\hline Locus tag & Protein name & Accession number & Fold-change & Protein function \\
\hline \multicolumn{5}{|c|}{ Up-regulated proteins $(50 \mathrm{rpm} / 150 \mathrm{rpm})$} \\
\hline PP_0234 & OprE & gi|26986977 & $2.41^{*}$ & Outer membrane porin \\
\hline PP_0268 & OprQ & gi|26987010 & 1.80 & Outer membrane porin \\
\hline PP_0465 & RplX & gi|26987206 & 1.61 & $50 S$ ribosomal protein $L 24$ \\
\hline PP_0812 & CyoA & gi|26987548 & 1.82 & Ubiquinol oxidase subunit 2 \\
\hline PP_0988 & GcvP-1 & gi|26987724 & 2.53 & Glycine dehydrogenase \\
\hline PP_1037 & PurL & gi|26987773 & $1.59^{*}$ & Phosphoribosylformylglycinamidine synthase \\
\hline PP_1099 & & gi|26987835 & 1.74 & Cold-shock domain-contain protein \\
\hline PP_1629 & $\operatorname{Rec} A$ & gi|26988361 & $2.35^{*}$ & Recombinase A \\
\hline PP_1868 & & gi|26988598 & $2.25^{*}$ & DEAD-box ATP dependent DNA helicase \\
\hline PP_1982 & $\mathrm{IbpA}$ & gi|26988708 & $8.33^{*}$ & Heat shock protein Hsp20 \\
\hline PP_2468 & RplT & gi|26989191 & 1.64 & $50 S$ ribosomal protein $L 20$ \\
\hline PP_2645 & MgtB & gi|26989364 & $2.67^{*}$ & Magnesium-translocating P-type ATPase \\
\hline PP_2656 & PstS & gi|26989375 & $4.26^{*}$ & Phosphate $A B C$ transporter, periplasmic phosphate-binding protein \\
\hline PP_4718 & $\mathrm{FtsH}$ & gi|26991401 & 2.04 & ATP-dependent metalloprotease FtsH \\
\hline PP_4803 & DacA & gi|26991483 & $1.96^{*}$ & Serine-type D-Ala-D-Ala carboxypeptidase \\
\hline PP_5329 & PstS & gi|26992005 & $3.33^{*}$ & Phosphate $A B C$ transporter phosphate-binding protein \\
\hline PP_0460 & & gi|24981839 & 1.65 & Ribosomal protein S3 \\
\hline \multicolumn{5}{|c|}{ Down-regulated proteins (50 rpm/150 rpm) } \\
\hline PP_0126 & & gi|26986871 & $0.37^{*}$ & Cytochrome c4 \\
\hline PP_0258 & & gi|26987000 & $0.21^{*}$ & Hypothetical protein PP_0258 \\
\hline PP_0296 & & gi|26987038 & $0.36^{*}$ & Glycine betaine/L-proline $A B C$ transporter, periplasmic binding protein \\
\hline PP_0308 & & gi|26987050 & 0.37 & Membrane dipeptidase \\
\hline PP_0315 & & gi|26987057 & 0.22 & Rieske (2Fe-2S) domain protein \\
\hline PP_0322 & GlyA-1 & gi|26987064 & 0.44 & Serine hydroxymethyltransferase \\
\hline PP_0328 & FdhA & gi|26987070 & $0.38^{*}$ & Formaldehyde dehydrogenase, glutathione-independent \\
\hline PP_0382 & & gi|26987124 & 0.41 & Nitrilase/cyanide hydratase and apolipoprotein $\mathrm{N}$-acyltransferase \\
\hline PP_0395 & & gi|26987137 & 0.19 & Hypothetical protein PP_0395 \\
\hline PP_0397 & & gi|26987139 & $0.28^{*}$ & Putative serine protein kinase, PrkA \\
\hline PP_0541 & & gi|26987279 & 0.28 & Acetyltransferase \\
\hline PP_0545 & & gi|26987283 & $0.43^{*}$ & Aldehyde dehydrogenase family protein \\
\hline PP_0763 & & gi|26987499 & 0.50 & Acyl-CoA synthetase \\
\hline PP_0765 & & gi|26987501 & $0.45^{*}$ & Hypothetical protein PP_0765 \\
\hline PP_0951 & RpoX & gi|26987687 & $0.34^{*}$ & Sigma 54 modulation protein/ribosomal protein S30EA \\
\hline PP_0999 & ArcC & gi|26987735 & $0.23^{*}$ & Carbamate kinase \\
\hline PP_1000 & Argl & gi|26987736 & $0.28^{*}$ & Ornithine carbamoyltransferase \\
\hline PP_1001 & ArcA & gi|26987737 & $0.24^{*}$ & Arginine deiminase \\
\hline PP_1015 & & gi|26987751 & 0.52 & Sugar $A B C$ transporter, periplasmic sugar-binding protein \\
\hline PP_1081 & & gi|26987817 & $0.44^{*}$ & Glutaredoxin-related protein \\
\hline PP_1084 & & gi|26987820 & 0.42 & Anti-oxidant AhpCTSA family protein \\
\hline PP_1122 & & gi|26987858 & 0.22 & OmpA/MotB domain protein \\
\hline PP_1210 & & gi|26987945 & $0.32^{*}$ & DNA-binding stress protein, putative \\
\hline PP_1478 & & gi|26988211 & $0.23^{*}$ & NADH:flavin oxidoreductase/NADH oxidase \\
\hline PP_1487 & & gi|26988220 & $0.40^{*}$ & Hypothetical protein PP_1487 \\
\hline
\end{tabular}


Table 1 Comparative proteome profile of $P$. putida grown at $50 \mathrm{rpm}$ and $150 \mathrm{rpm}$ (Continued)

\begin{tabular}{|c|c|c|c|c|}
\hline PP_1506 & Adk & gi|26988238 & $0.34^{*}$ & Adenylate kinase \\
\hline PP_1661 & & gi|26988393 & $0.41^{*}$ & Dehydrogenase subunit, putative \\
\hline PP_1741 & & gi|26988472 & $0.28^{*}$ & Substrate-binding region of ABC-type glycine betaine transport system \\
\hline PP_1859 & Ohr & gi|26988589 & $0.16^{*}$ & OsmC family protein \\
\hline PP_2006 & & gi|26988731 & $0.12^{*}$ & Hypothetical protein PP_2006 \\
\hline PP_2105 & & gi|26988830 & 0.48 & Hypothetical protein PP_2105 \\
\hline PP_2112 & AcnA & gi|26988836 & $0.42^{*}$ & Aconitate hydratase \\
\hline PP_2140 & & gi|26988864 & 0.47 & Hypothetical protein PP_2140 \\
\hline PP_2303 & HupB & gi|26989027 & 0.52 & Histone family protein DNA-binding protein \\
\hline PP_3089 & & gi|26989808 & $0.37^{*}$ & Hypothetical protein PP_3089 \\
\hline PP_3232 & & gi|26989950 & $0.16^{*}$ & Acetyltransferase \\
\hline PP_3283 & PhaB & gi|26990001 & $0.21^{*}$ & Enoyl-CoA hydratase \\
\hline PP_3433 & $\mathrm{Hpd}$ & gi|26990146 & $0.25^{*}$ & 4-hydroxyphenylpyruvate dioxygenase \\
\hline PP_3611 & & gi|26990322 & $0.12^{*}$ & Hypothetical protein PP_3611 \\
\hline PP_3668 & & gi|26990379 & $0.28^{*}$ & Catalase/peroxidase HPI \\
\hline PP_3765 & & gi|26990470 & $0.24^{*}$ & Transcriptional regulator MvaT, P16 subunit, putative \\
\hline PP_3839 & AdhA & gi|26990544 & $0.30^{*}$ & Alcohol dehydrogenase \\
\hline PP_4011 & Icd & gi|26990716 & $0.25^{*}$ & Isocitrate dehydrogenase, NADP-dependent \\
\hline PP_4034 & & gi|26990737 & $0.38^{*}$ & Allantoate amidohydrolase \\
\hline PP_4037 & & gi|26990739 & $0.32^{*}$ & Putative oxidoreductase \\
\hline PP_4038 & & gi|26990740 & $0.26^{*}$ & Dihydropyrimidine dehydrogenase \\
\hline PP_4116 & AceA & gi|26990810 & $0.27^{*}$ & Isocitrate lyase \\
\hline PP_4486 & & gi|26991172 & 0.51 & Cationic amino acid ABC transporter, periplasmic binding protein \\
\hline PP_4490 & PhhA & gi|26991176 & $0.47^{*}$ & Phenylalanine 4-monooxygenase \\
\hline PP_4593 & & gi|26991277 & $0.20^{*}$ & Hypothetical protein PP_4593 \\
\hline PP_4666 & MmsB & gi|26991350 & $0.24^{*}$ & 3-hydroxyisobutyrate dehydrogenase \\
\hline PP_4667 & MmsA-2 & gi|26991351 & $0.28^{*}$ & Methylmalonate-semialdehyde dehydrogenase \\
\hline PP_4848 & & gi|26991528 & 0.54 & DnaJ family curved-DNA-binding protein \\
\hline PP_4870 & & gi|26991550 & $0.38^{*}$ & Azurin \\
\hline PP_5007 & & gi|26991684 & $0.33^{*}$ & Poly(hydroxyalkanoate) granule-associated protein \\
\hline PP_5220 & ElbB & gi|26991896 & 0.45 & Isoprenoid biosynthesis protein \\
\hline PP_5232 & & gi|26991908 & 0.48 & Hypothetical protein PP_5232 \\
\hline PP_5258 & & gi|26991934 & $0.27^{*}$ & Aldehyde dehydrogenase family protein \\
\hline PP_5260 & & gi|26991936 & $0.24^{*}$ & Hypothetical protein PP_5260 \\
\hline
\end{tabular}

${ }^{*}$ P-value $<0.05$.

\section{Discussion}

As a soil organism, $P$. putida recurrently encounters filament-inducing conditions during its natural life cycle. Our data indicate that filament formation of $P$. putida could confer environmentally advantageous traits. Indeed, P. putida KT2440 grown at low shaking speed produced filaments and was more resistant to heat shock and saline stress. Similar observations were made for Caulobacter crescentus filaments, which showed a higher resistance to oxidative, osmotic, thermal and acid stress [18].

The comparative proteome profile indicated that the metabolic activity of $P$. putida KT2440 grown at $50 \mathrm{rpm}$ was significantly different from $P$. putida KT2440 grown at $150 \mathrm{rpm}$. The most pronounced induction occurred for the heat shock protein IbpA. This small heat shock protein belongs to the widely conserved family of $\alpha$-crystallin-type heat shock proteins. The latter appears to play 
a very versatile role in the protection against different stress conditions via protein and membrane protection [19]. In addition, many small heat shock proteins form oligomers, which may vary by the degree of phosphorylation or ion concentration [20] (induction of PP_2645, PP_2656 and PP_5329).

Although no observable differences in dissolved oxygen levels could be reported at the time of proteomic analysis (i.e., 15 hours, below detection limit for both conditions) (Figure 2), this does not completely rule out the role of dissolved oxygen in the observed results as the maximum oxygen transfer rate at $150 \mathrm{rpm}$ is approximately 2.5 times higher than at $50 \mathrm{rpm} \mathrm{[15].} \mathrm{Ohr,}$ a protein of the OsmC family (osmotically inducible protein) was 6.25-fold down-regulated in filamented $P$. putida, and is involved in the resistance to oxidative stressors, such as organic peroxide, but not in osmotic stress resistance [21]. In addition to a decreased Ohr abundance, other proteins involved in oxidative stress resistance were present at lower levels in $50 \mathrm{rpm}$ samples, including a catalase/peroxidase (PP_3668, 0.28-fold), an antioxidant AhpC (PP_1084, 0.42-fold), a glutaredoxin-related protein (PP_1081, 0.44 fold) and a putative DNA binding stress protein (PP_1210, 0.32-fold). The latter has recently been described as an oxidative stress-inducible Dps miniferritin $[22,23]$, and was found up-regulated in an OxyR mutant of $P$. aeruginosa [23]. The differential abundance of proteins involved in oxidative stress resistance could potentially be explained by lower oxygen levels in $50 \mathrm{rpm}$ cultures (and/ or decreased catabolism). The increase of OprE (PP_0234, 2.41-fold) and CyoA (PP_0812, 1.82-fold) further suggests limitations in oxygen availability in $50 \mathrm{rpm}$ cultures $[24,25]$. Finally, oxygen limitation is related to bacterial filamentation and/or RecA induction [6,26-28]. However, Jenssen and colleagues determined that, in addition to oxygen deprivation, additional factors such as exhaustion of medium components and changes in growth rate, were important for P. putida filamentation [6].

While RecA was more abundant in P. putida KT2440 grown at $50 \mathrm{rpm}$, the $P$. putida KT2440 recA mutant filamented at similar levels as the wild type. A similar observation was reported previously, showing that an E. coli recA mutant displayed similar levels of filamentation as the wild type strain in response to growth at high pressure, despite strong evidence of RecA-mediated SOS response activation [29-31]. Gottesman et al. (1981) suggested the existence of a transient filamentation phenotype in response to UV, independent of SulA [32], which could explain the RecAindependent filamentation phenotype of 50 rpm-grown $P$. putida KT2440 in the present study.

While the bacterial SOS response and associated filamentation is typically triggered by treatments directly affecting DNA integrity (e.g. exposure to mitomycin $\mathrm{C}$ or UV), a number of environmental conditions were reported to cause DNA damage in an indirect manner (e.g. starvation, aging, $\beta$-lactam antibiotics and high pressure stress) [30,33-36]. As such, high pressure-induced filamentation of $E$. coli was shown to stem from the activation of a cryptic Type IV restriction endonuclease (i.e. Mrr) endogenously present in the cell [37], while $\beta$-lactam antibiotics triggered DpiA to interfere with DNA replication [30,36]. Even though it remains unclear which metabolic changes could indirectly lead to DNA damage and SOS response activation, the major changes in metabolism provide evidence for new triggers of the SOS response.

\section{Conclusion}

In conclusion, our data indicate that filament-formation of $P$. putida KT2440 could confer environmentally advantageous traits, by increasing its resistance to saline and heat shock. We demonstrated that culturing at low shaking speed induced expression of RecA, which plays a central role in the SOS response, putatively through changes in amino acid metabolism and/or oxygen availability. Furthermore, the increased heat shock resistance was found to be RecA dependent. Filamentation could thus represent an adaptive survival strategy of $P$. putida, allowing it to persist during times of elevated soil temperatures, increased osmolarity (e.g., due to soil water evaporation) and/or increased pollution.

\section{Methods}

\section{Bacterial strains, media and growth conditions}

P. putida KT2440 (ATCC 12633) and its isogenic recA mutant derivative (kindly provided by Juan-Luis Ramos) were used in the present study. The bacterial strains were grown in Luria Bertani (LB) medium at $30^{\circ} \mathrm{C}$. For incubation at different shaking speeds, an overnight shaking culture $(150 \mathrm{rpm})$ of $P$. putida was diluted $100 \mathrm{x}$ in fresh LB medium. Ten milliliters of the dilution were transferred into $50 \mathrm{ml}$ Erlenmeyer flasks. The flasks were placed on an orbital shaker at $50 \mathrm{rpm}$ (filament-inducing condition) or at $150 \mathrm{rpm}$ (non-filament-inducing condition) [6]. Growth was monitored by measuring optical density at the $600 \mathrm{~nm}\left(\mathrm{OD}_{600}\right)$.

\section{Analysis of dissolved oxygen levels}

The measurement of the dissolved oxygen (DO) concentration of 50 and $150 \mathrm{rpm}$ cultures was performed using a Knick KNI913 oxygen meter. DO levels were measured during culture, at $15 \mathrm{~min}$ intervals for 24 hours.

\section{Environmental stress assays}

The assessment of cell viability following exposure to saline, acid and thermal stress was performed on P. putida KT2440 grown at 50 and $150 \mathrm{rpm}$ for 15 hours as described previously [38]. The concentrations of each stress agent were as follows: $5 \% \mathrm{NaCl}$ for osmotic stress 
and $10^{-4} \mathrm{M}$ citric acid for acid stress resistance $(\mathrm{pH}=5)$. For heat shock, exposure of cultures to a temperature of $55^{\circ} \mathrm{C}$ was applied. Cells were exposed to each stress for 30 minutes. Bacteria were diluted and plated on LB agar before and after exposure to the stress factors in order to determine the survival percentage.

\section{Bacterial morphology}

The morphology of $P$. putida KT2440 following incubation at different shaking speeds was visualized by fluorescence microscopy of Hoechst stained cells. Briefly, $600 \mu \mathrm{l}$ of bacterial culture (after 15 hours of growth) was resuspended in $500 \mu \mathrm{l} 70 \%$ ethanol to fix the cells, incubated at room temperature for $20 \mathrm{~min}$ and resuspended in saline solution. Next, $2.5 \mu$ l Hoechst solution $(200 \mu \mathrm{g} / \mathrm{ml}$ ) (Hoechst 33258, Sigma-Aldrich, Belgium) was added and incubated for 20 min. Five microliters of this suspension was transferred to a microscopic glass slide, covered with a coverslip and analyzed with a Zeiss Axiovert 100M fluorescence microscope (350 nm filter, 100x oil objective). Acquisition of images was performed with an Axiocam and further processed using the Axiovision software package.

\section{Flow cytometry analysis}

P. putida KT2440 grown at different shaking speeds was analyzed with an Accuri C6 flow cytometer (Accuri Cytometers) to assess the average cell length. Forward and side scatter signals were measured and a total of at least 10,000 cells were recorded for each sample. The respective cell populations were delimited to eliminate background signals originating from cell debris. All data analysis was performed with the CFlow Software.

\section{Proteomics}

Protein extraction and analysis was performed on $P$. putida grown at 50 and $150 \mathrm{rpm}$ for 15 hours. Proteins were extracted and labeled isotopically using ICPL, and the post-digest procedure was performed as described in [39]. Labeled tryptic peptides were submitted to online 2D-LC separation prior to MS/MS analysis as described previously [39], except that SCX column was eluted with 11 plugs of increasing $\mathrm{NH}_{4} \mathrm{Cl}$ concentration $(5,10,25$, $50,75,100,125,150,200,400$ and $800 \mathrm{mM}$ in loading solvent).

For MS/MS data processing, peptide peaks were detected and processed using Mascot Distiller (version 2.3.2). Created peak list was used as the input for Mascot MS/MS Ions searches using an in-house Mascot 2.2 server (Matrix Science) against the NCBInr database restricted to Pseudomonas putida (KT2440). The search parameters used were: enzyme $=$ trypsin; Max. Missed cleavages $=2$; Fixed modifications $=$ Carbamidomethyl $(C)$; Variable mod ifications = Oxidation (M); ICPL modification at both peptide $\mathrm{N}$-ter and lysine side chain. Peptide tolerance $\pm 1.3 \mathrm{Da}$;
MS/MS tolerance $\pm 0.5 \mathrm{Da}$; Peptide charge $=2+$ and $3+$; Instrument $=$ ESI-TRAP. Only proteins identified with a protein score above the calculated Mascot ion score, defined as the $95 \%$ confidence level, were considered.

Mascot distiller was also used for protein quantification with parameters as follows: integration method: simple; correlation threshold: 0.8 ; standard error threshold: 999; Xic threshold: 0.2; max Xic width: 7; fraction threshold: 0.5 and mass time matches allowed. Only peptides with an ion score above 30 were considered for quantification. The protein ratio corresponds to the average of peptide ratios. After examination that the distribution of protein ratios was almost centered on 1, a normalization based on the median of the peptide ratios was realized by mascot distiller on the complete dataset. Proteins with fold changes above 1.5 or below 0.66 were considered as in modified abundance.

\section{Statistical analysis}

All experiments were performed in triplicate, unless stated otherwise. The statistical determination of significance $(\alpha=0.05)$ was calculated using a Student's $t$-test on the biological replicates of each experimental condition.

\section{Additional files}

\begin{abstract}
Additional file 1: Figure S1. Morphologic analysis of a P. putida KT2440 isogenic recA mutant grown at $50 \mathrm{rpm}$ and $150 \mathrm{rpm}$. Flow cytometry dot plot (forward scatter versus side scatter) of $P$. putida KT2440 recA mutant grown at $50 \mathrm{rpm}$ (A) and $150 \mathrm{rpm}$ (B). Microscopic imaging of Hoechststained $P$. putida KT2440 recA mutant grown at $50 \mathrm{rpm}$ (C) and $150 \mathrm{rpm}$ (D) (magnification $=1000 \mathrm{x}$ ). Flow cytometry histogram of $P$. putida KT2440 recA mutant grown at $50 \mathrm{rpm}$ (grey line) and $150 \mathrm{rpm}$ (black line) (E), representing the average bacterial length.
\end{abstract}

Additional file 2: Figure S2. 3 Heat shock resistance of a P. putida KT2440 isogenic recA mutant grown at 50 and $150 \mathrm{rpm}$, as compared to wild type. Bacteria were exposed to $55^{\circ} \mathrm{C}$ during $30 \mathrm{~min}$.

\section{Competing interest}

The authors declare that no competing interests exist.

\section{Authors' contributions}

$\mathrm{AC}$ and RVH designed the study; contributed to the acquisition, analysis and interpretation of data, and wrote the manuscript. BL and RW performed proteomic analysis and data interpretation. AA assisted in data interpretation and contributed to manuscript writing. PC contributed to data interpretation, and NL helped to draft the manuscript. All authors read and approved the final manuscript.

\section{Acknowledgements}

This work was partially supported by the European Space Agency ESA/ESTEC through the PRODEX program in collaboration with the Belgian Science Policy through the BASE project. We thank Ilse Coninx, Wietse Heylen and Giuseppe Pani for excellent technical assistance.

\section{Author details}

${ }^{1}$ Unit of Microbiology, Expert Group Molecular and Cellular Biology, Institute for Environment, Health and Safety, Belgian Nuclear Research Centre (SCK (EN), Mol, Belgium. ²Department of Proteomics and Microbiology, Interdisciplinary Center of Mass Spectrometry (CISMa), University of Mons (UMONS), Mons, Belgium. ${ }^{3}$ Laboratory of Food Microbiology and Leuven Food Science and Nutrition Research Centre, Centre for Food and Microbial 
Technology, Department of Microbial and Molecular Systems, Faculty of Bioscience Engineering, Katholieke Universiteit Leuven, Leuven, Belgium. ${ }^{4}$ Laboratory of Microbial Interactions, Department of Molecular and Cellular Interactions, Flanders Institute for Biotechnology (VIB), Vrije Universiteit Brussel, Brussels, Belgium. ${ }^{5}$ Present address: The Biodesign Institute, Center for Infectious Diseases and Vaccinology, Arizona State University, 1001 S. McAllister Avenue, Tempe, AZ 85287, USA.

Received: 22 June 2012 Accepted: 16 November 2012

Published: 27 November 2012

\section{References}

1. Wu X, Monchy S, Taghavi S, Zhu W, Ramos J, van der Lelie D: Comparative genomics and functional analysis of niche-specific adaptation in Pseudomonas putida. FEMS Microbiol Rev 2011, 35(2):299-323.

2. Dixon RA: Natural products and plant disease resistance. Nature 2001, 411(6839):843-847.

3. Manzanera M, Aranda-Olmedo I, Ramos JL, Marques S: Molecular characterization of Pseudomonas putida KT2440 rpoH gene regulation. Microbiology 2001, 147(Pt 5):1323-1330.

4. Srivastava S, Yadav A, Seem K, Mishra S, Chaudhary V, Nautiyal CS: Effect of high temperature on Pseudomonas putida NBRI0987 biofilm formation and expression of stress sigma factor RpoS. Curr Microbiol 2008, 56(5):453-457

5. Tegos G, Stermitz FR, Lomovskaya O, Lewis K: Multidrug pump inhibitors uncover remarkable activity of plant antimicrobials. Antimicrob Agents Chemother 2002, 46(10):3133-3141.

6. Jensen $\mathrm{RH}$, Woolfolk CA: Formation of filaments by Pseudomonas putida. Appl Environ Microbiol 1985, 50(2):364-372.

7. Justice SS, Hunstad DA, Cegelski L, Hultgren SJ: Morphological plasticity as a bacterial survival strategy. Nat Rev Microbiol 2008, 6(2):162-168.

8. Boberek JM, Stach J, Good L: Genetic evidence for inhibition of bacterial division protein FtsZ by berberine. PLoS One 2010, 5(10):e13745.

9. Mattick KL, Jorgensen F, Legan JD, Cole MB, Porter J, Lappin-Scott HM, Humphrey TJ: Survival and filamentation of Salmonella enterica serovar Enteritidis PT4 and Salmonella enterica serovar Typhimurium DT104 at low water activity. Appl Environ Microbiol 2000, 66(4):1274-1279.

10. Shaw MK: Formation of filaments and synthesis of macromolecules at temperatures below the minimum for growth of Escherichia coli. J Bacteriol 1968, 95(1):221-230.

11. Steinberger RE, Allen $A R$, Hansa HG, Holden PA: Elongation correlates with nutrient deprivation in Pseudomonas aeruginosa-unsaturates biofilms. Microb Ecol 2002, 43(4):416-423.

12. Chang WS, Halverson LJ: Reduced water availability influences the dynamics, development, and ultrastructural properties of Pseudomonas putida biofilms. J Bacterio/ 2003, 185(20):6199-6204.

13. Justice SS, Hung C, Theriot JA, Fletcher DA, Anderson GG, Footer MJ, Hultgren SJ: Differentiation and developmental pathways of uropathogenic Escherichia coli in urinary tract pathogenesis. Proc Natl Acad Sci USA 2004, 101(5):1333-1338.

14. Rosenberger CM, Finlay BB: Macrophages inhibit Salmonella typhimurium replication through MEK/ERK kinase and phagocyte NADPH oxidase activities. J Biol Chem 2002, 277(21):18753-18762.

15. Maier U, Buchs J: Characterisation of the gas-liquid mass transfer in shaking bioreactors. Biochem Eng J 2001, 7(2):99-106.

16. Michel B: After 30 years of study, the bacterial SOS response still surprises us. PLOS Biol 2005, 3(7):e255

17. McCool JD, Long E, Petrosino JF, Sandler HA, Rosenberg SM, Sandler SJ: Measurement of SOS expression in individual Escherichia coli K-12 cells using fluorescence microscopy. Mol Microbiol 2004, 53(5):1343-1357.

18. Wortinger MA, Quardokus EM, Brun W: Morphological adaptation and inhibition of cell division during stationary phase in Caulobacter crescentus. Mol Microbiol 1998, 29(4):963-973.

19. Narberhaus F: Alpha-crystallin-type heat shock proteins: socializing minichaperones in the context of a multichaperone network. Microbiol Mol Biol Rev 2002, 66(1):64-93. table of contents.

20. Kato K, Hasegawa K, Goto S, Inaguma Y: Dissociation as a result of phosphorylation of an aggregated form of the small stress protein, hsp27. J Biol Chem 1994, 269(15):11274-11278.

21. Atichartpongkul S, Loprasert S, Vattanaviboon P, Whangsuk W, Helmann JD, Mongkolsuk S: Bacterial Ohr and OsmC paralogues define two protein families with distinct functions and patterns of expression. Microbiology 2001, 147(Pt 7):1775-1782

22. Bellapadrona G, Ardini M, Ceci P, Stefanini S, Chiancone E: Dps proteins prevent Fenton-mediated oxidative damage by trapping hydroxyl radicals within the protein shell. Free Radic Biol Med 2010, 48(2):292-297.

23. Vinckx T, Wei Q, Matthiis S, Noben JP, Daniels R, Cornelis P: A proteome analysis of the response of a Pseudomonas aeruginosa oxyR mutant to iron limitation. Biometals 2011, 24(3):523-532.

24. Williams HD, Ziosnik JEA, Ryall B: Oxygen, cyanide and energy generation in the cystic fibrosis pathogen Pseudomonas aeruginosa. Adv Microb Physiol 2007, 52:1-71.

25. Yamano $Y$, Nishikawa T, Komatsu Y: Involvement of the RpoN protein in the transcription of the oprE gene in Pseudomonas aeruginosa. FEMS Microbiol Lett 1998, 162(1):31-37.

26. Filiatrault MJ, Wagner VE, Bushnell D, Haidaris CG, Iglewski BH, Passador L: Effect of anaerobiosis and nitrate on gene expression in Pseudomonas aeruginosa. Infect Immun 2005, 73(6):3764-3772.

27. Nishimura T, Teramoto $H$, Inui M, Yukawa $H$ : Gene expression profiling of Corynebacterium glutamicum during anaerobic nitrate respiration: induction of the SOS response for cell survival. J Bacteriol 2011, 193(6):1327-1333.

28. Sellars MJ, Hall SJ, Kelly DJ: Growth of Campylobacter jejuni supported by respiration of fumarate, nitrate, nitrite, trimethylamine- $N$-oxide, or dimethyl sulfoxide requires oxygen. J Bacteriol 2002, 184(15):4187-4196.

29. Aertsen A, Michiels CW: SulA-dependent hypersensitivity to high pressure and hyperfilamentation after high-pressure treatment of Escherichia coli Ion mutants. Res Microbiol 2005, 156(2):233-237.

30. Aertsen A, Van Houdt R, Vanoirbeek K, Michiels CW: An SOS response induced by high pressure in Escherichia coli. J Bacteriol 2004 186(18):6133-6141.

31. Kawarai T, Wachi M, Ogino H, Furukawa S, Suzuki K, Ogihara H, Yamasaki M: SulA-independent filamentation of Escherichia coli during growth after release from high hydrostatic pressure treatment. Appl Microbiol Biotechnol 2004, 64(2):255-262.

32. Gottesman $S$, Halpern $E$, Trisler P: Role of sulA and sulB in filamentation by Ion mutants of Escherichia coli K-12. J Bacteriol 1981, 148(1):265-273.

33. Aertsen A, Michiels CW: Upstream of the SOS response: figure out the trigger. Trends Microbiol 2006, 14(10):421-423.

34. Bjedov I, Tenaillon O, Gerard B, Souza V, Denamur E, Radman M, Taddei F, Matic I: Stress-induced mutagenesis in bacteria. Science 2003, 300(5624):1404-1409.

35. Humayun MZ: SOS and Mayday: multiple inducible mutagenic pathways in Escherichia coli. Mol Microbiol 1998, 30(5):905-910

36. Miller C, Thomsen LE, Gaggero C, Mosseri R, Ingmer H, Cohen SN: SOS response induction by beta-lactams and bacterial defense against antibiotic lethality. Science 2004, 305(5690):1629-1631.

37. Aertsen A, Michiels CW: Mrr instigates the SOS response after high pressure stress in Escherichia coli. Mol Microbiol 2005, 58(5):1381-1391.

38. Crabbé A, Pycke B, Van Houdt R, Monsieurs P, Nickerson C, Leys N, Cornelis $\mathrm{P}$ : Response of Pseudomonas aeruginosa PAO1 to low shear modelled microgravity involves AlgU regulation. Environ Microbiol 2010, 12(6):1545-1564.

39. Leroy B, Rosier C, Erculisse V, Leys N, Mergeay M, Wattiez R: Differential proteomic analysis using isotope-coded protein-labeling strategies: comparison, improvements and application to simulated microgravity effect on Cupriavidus metallidurans CH34. Proteomics 2010, 10(12):2281-2291.

doi:10.1186/1471-2180-12-282

Cite this article as: Crabbé et al:: Differential proteomics and physiology of Pseudomonas putida KT2440 under filament-inducing conditions. BMC Microbiology 2012 12:282. 